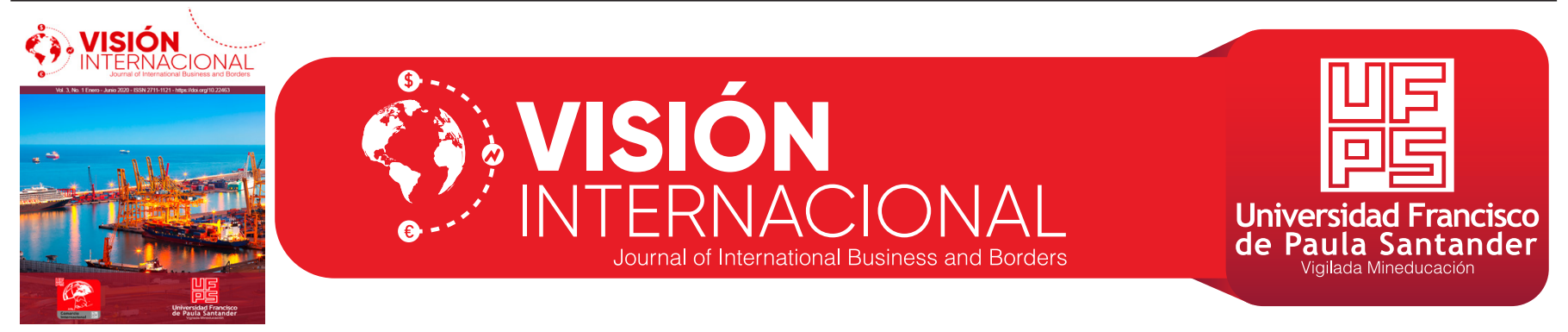

Artículo Original

https://doi.org/

\title{
Implementación de las Industrias 4.0 como Componente Digital Para La Transformación Digital de la Economía de Norte De Santander.
}

Implementation of Industries 4.0 as a Digital Component for the Digital Transformation of the Economy of Norte De Santander.

Pablo Emilio Ballesteros-Peñaranda ${ }^{1}$, Shirley Andrea Gómez-González², Valeryth Jazmín Mendoza-Franco

'pabloemiliobp@ufps.edu.co

${ }^{2}$ shirleyandreagg@ufps.edu.co

Valerythfranco@gmail.com

Cómo citar: P.E. Ballesteros-Peñaranda, S.A. Gómez-González, V.J. Mendoza-Franco (2020), "Implementación de las Industrias 4.0 como Componente Digital Para La Transformación Digital de la Economía de Norte De Santander.". Visión Interncional, vol. 3, no. 1, 19-30.

Recibido: Octubre 1,2019; Aprobado Diciembre 17,2019.

\begin{tabular}{ll}
\hline & RESUMEN \\
\hline Palabras Clave: & $\begin{array}{l}\text { En definitiva, la cuarta revolución industrial apenas comienza, investigar el tema en bases de datos de investigación } \\
\text { o internet trae definiciones similares; es un tema que se desarrolla con mayor fuerza en las organizaciones. }\end{array}$ \\
transformación digital, & $\begin{array}{l}\text { Actualmente, se investiga con mayor énfasis en este tema innovador, es por esto que se toma en cuenta una } \\
\text { variedad de perspectivas que giran alrededor del mundo digital y físico. En este momento, es difícil conceptualizar }\end{array}$ \\
industrias 4.0, & lo que esto significa. Cuando se aborda el concepto de innovación a través del lente de lo digital y lo físico, \\
componentes digitales, & significa repensar temas tan diversos como las ciudades, el trabajo, el dinero, el ocio y el entretenimiento. Y luego, \\
estrategias, & fundamentalmente, las personas buscan adaptarse a una nueva revolución industrial que crece rápidamente en un \\
innovación & mundo globalizado.
\end{tabular}

machine learning.

Eventualmente, siempre que se habla de transformación digital, se toma en cuenta los alcances positivos, pero también se posee retos que si no se controlan desde ahora puede que en el futuro se compliquen, en este caso me refiero a la caída de la crisis climática y la demografía creciente, que son factores causantes de las pasadas revoluciones industrias y que afecta a largo plazo la economía mundial. Es importante mencionar que el objetivo de estudio consiste en analizar los distintos estudios de industrias 4.0, economía y transformación digital, todo esto con el fin de implementar estrategias de superación y adquisición de las industrias 4.0 en las pymes cucuteñas.

\begin{tabular}{ll}
\hline Key Words: & ABSTRACT \\
\hline $\begin{array}{l}\text { In short, the fourth industrial revolution is just beginning, researching the subject in research databases or the } \\
\text { internet brings similar definitions; It is a theme that develops with greater force in organizations. Currently, } \\
\text { industries 4.0, } \\
\text { digital components, } \\
\text { strategies, } \\
\text { innovation }\end{array}$ & $\begin{array}{l}\text { around the digital and physical world are taken into account. Right now, it is difficult to conceptualize what this } \\
\text { means. When the concept of innovation is approached through the lens of the digital and the physical, it means } \\
\text { rethinking issues as diverse as cities, work, money, leisure and entertainment. And then, fundamentally, people } \\
\text { machine learning. }\end{array}$ \\
& $\begin{array}{l}\text { Eventually, whenever we talk about digital transformation, the positive outcomes are taken into account, but } \\
\text { there are also challenges that, if not controlled from now on, may become more complicated in the future, in this } \\
\text { case I am referring to the fall of the climate crisis and growing demographics, which are factors causing the past } \\
\text { industrial revolutions and which affect the world economy in the long term. It is important to mention that the } \\
\text { objective of the study is to analyze the different studies of industries 4.0, economy and digital transformation, } \\
\text { all this in order to implement strategies for overcoming and acquiring industries } 4.0 \text { in SMEs Cucuteñas. }\end{array}$ \\
\hline
\end{tabular}

*Autor de correspondencia.

E-mail: pabloemiliobp@ufps.edu.co (Pablo Emilio Ballesteros-Peñaranda) 


\section{Introducción}

Al abordar el tema de la cuarta revolución industrial, se suele hablar en primera instancia de la imparable automatización que se aplicará a casi todas las tareas productivas y por lo tanto, de una inevitable ola de desempleo que está automatización provocará (Lirio,2020). La Industria 4.0, busca en sí, transformar a las empresas en organizaciones inteligentes con oportunidades de negocio. El alcance de la investigación logra atribuir a que componentes digitales son potenciales para la aplicación de industrias 4.0 en las organizaciones.

La implantación global de internet como una gran base de datos siempre disponibles ha permitido construir la imagen del mundo en que vivimos a partir de todos esos datos. Así, la tradicional inteligencia artificial, una disciplina que durante el siglo pasado parecía algo únicamente propio de laboratorios universitarios y de revistas de ciencia ficción, ha evolucionado hacia la cotidianidad bajo nuevos nombres y etiquetas, como pueden ser el caso del machine learning. Actualmente las organizaciones tienen la obligación de implementar nuevas tecnologías en su plan estratégico ya que todo esto depende del éxito en el marco de su participación en la competitividad global.

Compañías tecnológicas cuya actividad y excelencia proviene de estos campos acaban siendo compañías de contenidos y acaban superando a gigantes históricos de los contenidos. Solo hace falta pensar, por ejemplo, en el caso de Netflix. Así pues, tener claro qué beneficios, retos y riesgos implica la inteligencia artificial es una tarea ineludible para cualquier persona responsable de una empresa $u$ organización que pretenda perdurar en su entorno y no ser archivada prematuramente en los armarios de la historia, es decir innovar y adaptarse a los cambios que el medio provoca y sobrevivir.

Ahora bien, muchas empresas a nivel mundial han optado por poner algoritmos con el objetivo de recolectar información a través de los usuarios de internet, entonces, si alguna vezle ofrecen un producto gratis, por ejemplo, un curso de inglés. significa que no es relativamente gratis, se representa por medio de un costo/beneficio en donde usted mismo eres el producto. Lo anterior es muy tradicional ya que se repite en la industria de los datos. Redes sociales, prensa digital y aplicaciones de comercio electrónico ofrecen todo tipo de servicios de manera gratuita. Esta gratuidad hace referencia estrictamente al no tener que abonar dinero, ya que, por lo general, se está entregando algo a cambio de poder disfrutar de esos servicios.

Es normal y frecuente que aquellos negocios que estén basados en la inteligencia artificial moderna, es decir, aquella que funciona a partir de la recolección de grandes cantidades de datos, aparezcan en el mercado disfrazadas de proveedores de servicios gratuitos. Cada vez que se acepta alguno de los famosos retos que aparecen periódicamente en las redes sociales, como, por ejemplo, publicar un listado de los países donde ha vivido; en ese caso, se está alimentando algún algoritmo de machine learning con datos de entrenamiento o de validación. El ser humano está ayudando a educar dichos algoritmos que acabarán siendo capaces de crear estudios muy precisos y valiosos. Todo aludiendo a un costo/beneficio implementado por las empresas.

\section{Metodologías}

La metodología utilizada en el desarrollo de este estudio tiene un enfoque de revisión bibliográfica y se utilizan como fuentes de información las bases de datos de la Biblioteca Eduardo Cote Lamus, documentos tales como: revistas científicas, repositorios y artículos científicos entre los criterios de búsqueda en este caso los componentes digitales.

Los criterios de búsqueda utilizados para la revisión documental fueron industrias 4.0 y comercio internacional. Es decir, describe los componentes digitales fundamentales para la transformación digital de las pymes cucuteñas, de igual manera, se describen los beneficios, componentes, retos 
y resultados que aporta la transformación digital enfocada en las industrias 4.0 en la economía de la ciudad de Cúcuta, Norte de Santander. Todo lo anterior con el objetivo de analizar los datos referentes a las palabras claves de la investigación: Industrias 4.0, componentes digitales y transformación digital. Es importante mencionar que también se tienen en cuenta los aportes en cifras o datos de la Cámara de comercio de Cúcuta, el índice de economía digital (IED) y el DANE, como fuentes primarias.

\section{Desarrollo}

Según el índice de Economía digital (IED), la transformación digital a nivel país crece aceleradamente, cada día más empresas adoptan modelos de negocio tecnológicos enfocados en industrias 4.0. Desde el año 2017 se ha analizado el impacto de la tecnología en las pequeñas, medianas y grandes empresas (Portafolio, 2018). A continuación, se presenta la adopción de tecnologías dividido por regiones.

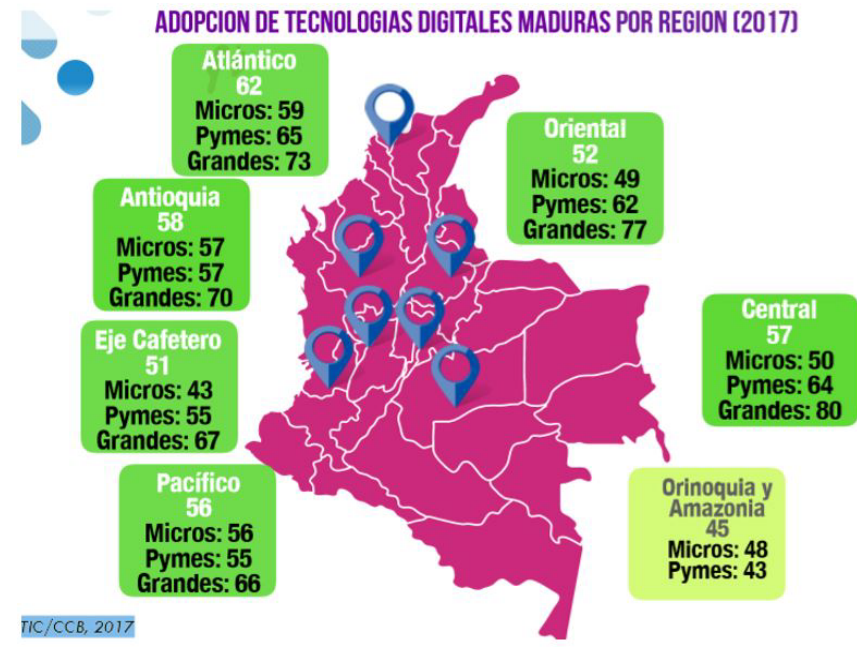

Figura 1. Adopción de tecnologías digitales maduras en Colombia (2017) Fuente: MINTIC/CCB, 2017

De manera que, como se logra observar en la (Figura 1), en la región oriental donde se encuentra el departamento de Norte de Santander varias empresas habían adoptado la tecnología en sus procesos, todo esto, es un buen comienzo ya que las organizaciones han implementado planes a largo plazo con el objetivo de sobrevivir a la cuarta revolución industrial.

La adopción por componente y tecnología muestra un avance importante en tecnologías que impactan procesos productivos. A continuación, se muestran datos de la adopción de los componentes digitales a nivel nacional en las pequeñas, grandes y medianas empresas.

Tabla I. Adopción de tecnologías en las empresas en Colombia

\begin{tabular}{|c|c|c|c|c|c|}
\hline Pilar & Tecnología & Nacional & Grandes & Pymes & Micros \\
\hline \multirow{3}{*}{ Infraestructura } & Ciberseguridad & $32.2 \%$ & $67.2 \%$ & $37.1 \%$ & $25.4 \%$ \\
\cline { 2 - 6 } & Computación en la nube & $19.1 \%$ & $48.8 \%$ & $22.9 \%$ & $13.6 \%$ \\
\hline \multirow{3}{*}{ Procesamiento } & Internet de las cosas & $9.0 \%$ & $14.8 \%$ & $9.3 \%$ & $8.2 \%$ \\
\cline { 2 - 6 } & Robótica & $1.5 \%$ & $11.1 \%$ & $1.2 \%$ & $0.6 \%$ \\
\cline { 2 - 6 } & Impresión 3D & $2.2 \%$ & $4.8 \%$ & $2.1 \%$ & $2.1 \%$ \\
\cline { 2 - 6 } & Realidad virtual & $1.0 \%$ & $1.7 \%$ & $0.9 \%$ & $1.0 \%$ \\
\hline \multirow{3}{*}{ Distribución } & Big data & $3.2 \%$ & $16.8 \%$ & $4.0 \%$ & $1.3 \%$ \\
\cline { 2 - 6 } & Inteligencia artificial & $1.8 \%$ & $9.7 \%$ & $2.4 \%$ & $0.7 \%$ \\
\cline { 2 - 6 } & Blockchain & $1.6 \%$ & $5.9 \%$ & $1.6 \%$ & $1.1 \%$ \\
\hline
\end{tabular}

Fuente: Encuesta MINTIC/CCB, 2017 
De la anterior tabla (Tabla 1) se deduce que, las grandes empresas están adoptando en mayor cantidad las tecnologías en sus procesos seguida por las pymes y las micro, En definitiva, se ha evidenciado que el factor predominante en las revoluciones industriales es la innovación, la evolución en los cambios tecnológicos describen la digitalización de los procesos de las empresas como una interconexión mediante internet de las cosas, todo esto en particular con el fin flexibilizar la individualización de los procesos de las empresas, aunque las tecnologías avanzadas dan soluciones más flexibles e inteligentes, en particular siempre se trata de automatizar las empresas tecnológicamente con el fin de suplir las necesidades del medio.

En el año 2017 se tuvo una adopción del 1.5\% de inteligencia artificial en las empresas de la región oriente del país (figura 2), además en la adopción de internet de las cosas tuvo una adopción del 2.2\% a $3.5 \%$ (figura 3 ) y por último la adopción en robótica alcanzó el 2.7\% (figura 4) (MinTic, 2017), como se muestra a continuación.

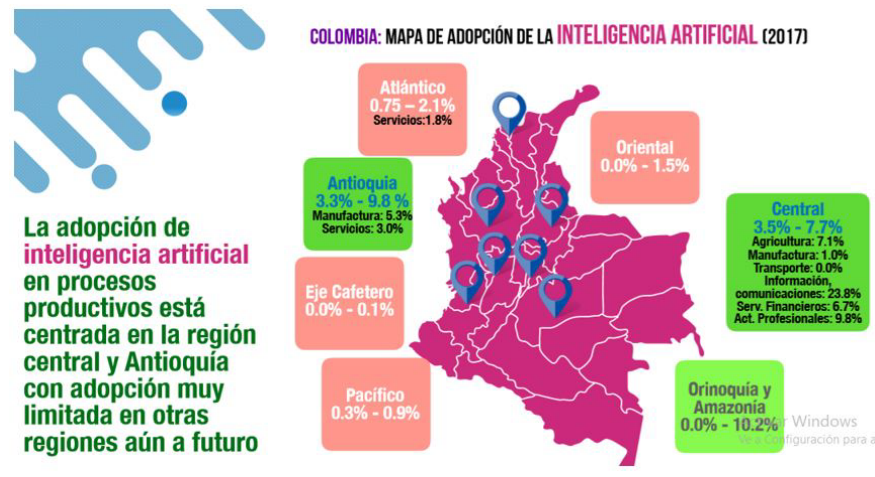

Figura 2. Adopción de inteligencia artificial en Colombia Fuente: Encuesta MINTIC/CCB, 2017

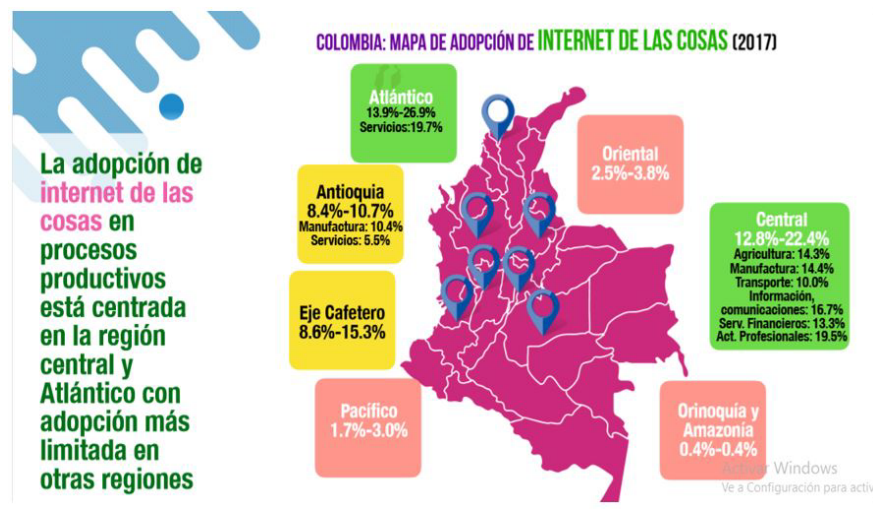

Figura 3. Adopción de internet de las cosas en Colombia Fuente: Encuesta MINTIC/CCB, 2017
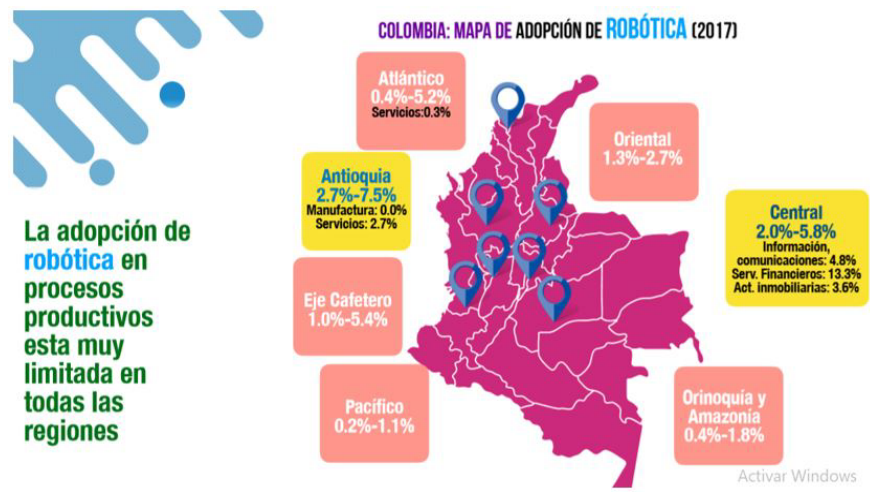

Figura 4. Adopción de la Robótica en Colombia Fuente: Encuesta MINTIC/CCB, 2017

En definitiva, la adopción de las industrias 4.0 es una oportunidad para las empresas en la nueva era digital, ya que la innovación es la palabra clave para transformarse, por ende, cada uno de los componentes digitales (machine learning, robotics, internet de las cosas entre otros) que se investigan son muy potenciales para su aplicación en los procesos de las empresas, todo esto con el objetivo de ser más competitivos en el mercado local, nacional e internacional y adaptarse a la cuarta revolución industrial. A continuación, se realiza la revisión bibliográfica de artículos de investigación enfocados en la transformación digital.

Factores de éxito de las industrias 4.0 en Guayaquil, Ecuador

Este artículo aporta a la investigación un diseño de estrategias de adaptabilidad a las industrias 4.0 y expone factores de éxito potenciales para su 
aplicación en las economías. Las empresas deben adaptarse a los cambios, en sí a la realidad, es decir, adoptar estrategias nuevas que les permita trabajar en conjunto con sus clientes en el proceso de diseño de los productos. Asimismo, la aplicación de nuevas tecnologías permite la creación o investigación sobre nuevos modelos de producción, en donde todos los procesos que conforman la empresa se comunican entre sí, de una forma más flexible, todo lo anterior con el fin de analizar la conexión de los productos con los distintos componentes digitales que en la actualidad se han vuelto exitosos, en última instancia, todo esto se denomina industrias 4.0 (Endesa, 2017).

Como resultado a causa de la baja productividad en ventas en el sector industrial de la ciudad de Guayaquil, con la falta de tecnología y capacitación de las empresas. Se realizó un estudio que tuvo un alcance para dotar de información necesaria a las industrias con una guía de estrategias de las industrias 4.0, con respecto a apoyar y ayudar en el proceso productivo de las empresas y la adaptación a las nuevas tecnologías. Todo lo anterior con el objetivo de minimizar el uso de materias primas e innovar, para así tener una mejor competitividad en la economía mundial (Reyes, 2019).

En síntesis, si las empresas se transforman bajo un modelo de industrias 4.0, una consecuencia de ellos primeramente son los altos costos de inversión, al momento que se adaptan a estos cambios las empresas, estas deben utilizar maquinaria automatizada además de los mantenimientos y la programación para que realicen cualquier tarea productiva. Segundo, la seguridad de la información, es un obstáculo para adaptarse a los nuevos cambios, ya que las empresas pueden ser víctimas de robos de información o alteración en sus procesos. Tercero, adaptabilidad al cambio tecnológico, ya que, si habla de esto, genera incertidumbre en las personas ya que su trabajo se reemplazará por un robot, sin embargo, estos cambios en realidad son esenciales para la sociedad. Finalmente, este estudio logró crear nuevas estrategias de investigación referentes a líneas como emprendimiento e innovación, producción, competitividad y desarrollo empresarial, todo lo anterior con el fin de brindar datos sobre el sector textil de Guayaquil, Ecuador y así generar iniciativa de investigación sobre el tema de industrias 4.0.

\section{EI papel del estado enfocado en la digitalización de la economía}

Este artículo aporta a la investigación un análisis de cómo los gobiernos hacen frente a las nuevas necesidades que surgen en la actualidad, entre ellos la economía digital que crece rápidamente, y está como contribuyente al PIB de un país, la economía digital se define como el uso de tecnologías ,cambios en el interior de las empresas, modelo innovador para mantener relación y contacto con los clientes, proveedores entre otros, de igual manera también se considera como un modelo de negocio exitoso que agrupa todo tipo de datos de localización y comportamientos que sirven para generar estrategias innovadoras en las empresas (Ballesteros, 2017). Concretamente en la investigación se analiza las distintas políticas que tienen los países frente a la economía digital y cómo los gobiernos pueden adaptarlas con el fin de flexibilizar al sector.

Así que, ante un mundo globalizado y dinámico, que lo conforman muchos actores entre ellos: compradores y vendedores, empresas, startups, investigadores, personas, ciudadanos en fin todas las personas hacemos parte de la economía mundial y jugamos un papel importante en ella. Así que los tiempos cambian y no es necesario mantener las mismas políticas de hace 20 años, es importante que los países incorporen una actitud que fortalezca la adaptación a los cambios y este sea eficiente para las empresas. Como resultado de todo lo anterior se analiza cómo el estado puede favorecer la adaptación a un nuevo entorno, anticipándose a los cambios y definir estrategias para así prepararse para recibir las oportunidades.

En resumen, las estrategias de los gobiernos para impulsar la economía digital se centran primeramente en implementar medidas que 
fomenten la economía digital en el país, facilitando la formación de nuevas empresas tecnológicas y transformarse como productor de servicios digitales, todo lo anterior con el objetivo de ser más competitivo a nivel mundial. Segundo, los gobiernos deben potencializar el sistema $\mathrm{I}+\mathrm{D}+\mathrm{i}$, no obstante apoyando a las organizaciones públicas o privadas en sus investigación entorno la economía digital, tercero adoptar medidas de modernizar el sector empresarial, incentivando la transformación digitales en las pymes y de este modo, fortalecer los emprendimientos de transformación digital atrayendo inversión extranjera directa y finalmente el desarrollo de una regulación inteligente, que sea clara con el objetivo de permitir un desarrollo ágil en las actividades digitales dentro de las empresas y el sector económico.

\section{Evaluación del desarrollo tecnológico para Industrias 4.0}

Este artículo aporta a la investigación un proyecto cuyo principal objetivo es identificar qué tipos TICs son potenciales para implementar en las diferentes industrias y cómo estas pueden utilizar estos componentes en sus procesos y áreas de negocio, de modo que, en la investigación se estudia la viabilidad de una aplicación software que permita analizar y evaluar las TICs en las empresas. Todo lo anterior se refiere a la posibilidad de conocer las distintas tecnologías que trae la aplicación de las industrias 4.0 en la transformación digital de las empresas, concretamente, para conseguir agregación de valor en la producción de las empresas (Mon, 2018).

En definitiva, el desarrollo de las TICs, favorece el desempeño de las organizaciones frente al cambio digital, la conectividad entre lo físico y lo digital y la resolución de problemas de la producción, es decir favorece todos los factores que se enfocan hacia lo que se conoce como industrias 4.0. El objetivo de la investigación es determinar específicamente cuáles son los avances tecnológicos que definen las industrias 4.0, de manera que se desarrolle un método de medición de inserción de TICs el cual permite determinar los tipos de productos de software que pueden ser potenciales para la incorporación en las industrias según su área funcional. Las industrias se dividen entre básico, medio y avanzado, las industrias que se encuentran nivel avanzado son las que están más desarrolladas tecnológicamente y pueden llegar a ser reconocidas como Industrias 4.0.

Finalmente, la investigación propone determinar qué tipos de TICSs puede contener las industrias y cómo estas pueden aportar para definirla en una industria 4.0 y así determinar qué criterios pueden cumplir las tecnologías con el fin de hacer más sencillo su uso para los usuarios en su contexto físico y digital.

\section{Digitalización y mercados de exportación}

Este artículo aporta a la investigación un análisis del impacto de la digitalización en la actualidad, puede ser un impacto positivo como negativo, en este caso es irrealizable determinar hacia dónde vamos, ya que se ha visto muchos eventos en los últimos años en la tecnología, todo esto corresponde a que solo estamos en el principio de una era digital (Villegas, 2017).

Como resultado en la relación entre la digitalización, las industrias 4.0 y el comercio internacional tiene como finalidad la creación potencial de nuevos mercados, un ejemplo de ello es la telemedicina con los robots Da Vinci, cuando un médico español puede operar en Francia sin moverse de su país y trasladarse, la digitalización ha logrado abaratar los costes, es decir a permitido la comercialización de los productos más fácilmente, de modo que las empresas se transformen en mercados preexistentes así como lo hizo la música y el cine, esta transformación implica la adaptación a modelos de negocio que faciliten la comercialización internacional de productos.

En definitiva, la digitalización en los mercados cambiará el mundo, el comercio, las sociedades, 
la política entre otros factores. Sin embargo, se determina como esta misma cambiara aun así a otras tecnologías. Hay muchas tecnologías dentro de la digitalización (Steve Jobs, 2007). En concreto los mercados deben innovar y adaptarse a los cambios que trae la digitalización y así reinventarse si el mundo cambiante lo requiere.

\section{Nuevos empleos, nuevas habilidades, ¿Estamos preparando el talento para la cuarta revolución industrial?}

Este artículo aporta a la investigación un análisis de cómo la cuarta revolución industrial está provocando una amenaza entre la demanda y los distintos profesionales y que estos cuenten con habilidades y capacidades que fortalezcan la Cuarta revolución industrial. A pesar de todo lo que se analiza a futuro es importante que las empresas y centros educativos establezcan un nuevo modelo educativo en donde se establezca la tecnología como principal fuente en desarrollo, todo esto son estrategias que ayudarán a adaptarse a los cambios (Pernías, 2017).

Como resultado, se analizó la cuarta revolución industrial va provocar mayor desigualdad(Kuzmenki, 2017). Es necesario, que las empresas cambien sus procesos y adapten tendencias que permitan preparar a los estudiantes y trabajadores para este nuevo escenario de economía mundial. Un ejemplo de ellos, lo adoptó Alemania en 2010, donde se propuso grandes cambios en los niveles educativos para enfrentar este tipo de cambios a largo plazo (Federal Ministry for Economics, 2010). En particular todo lo anterior es efecto del pronóstico del incremento de la desigualdad por efecto de la cuarta revolución industrial.

En conclusión, si las empresas no piensan en evolucionar ante los cambios que se vienen y solo se centra en su éxito a corto plazo, no será posible su rendimiento a largo plazo, en relación con la contratación de su capital humano, si la estrategia se trata de resolver solo problemas en el presente y llegue el momento de resolver los obstáculos y problemas futuros verán su compromiso con la competitividad.

\section{Utilización de machine learning en la industria 4.0}

En este estudio se resalta una introducción al machine learning bajo el uso y aplicación de las industrias 4.0, no obstante la utilidad del machine learning en el área productiva, de calidad, logística, mantenimiento, negocios y marketing de las empresas ayudan a suplir la demanda y la competitividad hacen que la aplicación de componentes digitales como machine learning o digitalización sea una oportunidad para las empresas. En definitiva, todas las organizaciones para sobrevivir deben adoptar planes tecnológicos e innovadoras en sus modelos de negocio para poder mantenerse en el mercado internacional (Maisueche, 2019).

Como resultado, la transformación digital supone distintos retos en cada uno de los sectores para grandes, medianas o pequeñas empresas. De modo que a pesar de identificar todo lo que ofrece el machine learning, aún no se ha experimentado todo su potencial.

En síntesis, el machine learning se entiende como una tecnología muy fundamental para las empresas, en efecto la capacidad del machine learning para tener información, es decir es potencial para el desarrollo de las empresas. Ahora bien, como líneas futuras, en la investigación se propone un estudio más detallado, en concreto, se trata de ampliar la aplicación del machine learning en los procesos de las empresas, ya que es importante la aplicación de tecnologías como machine learning de forma muy diferente para cubrir las necesidades que el mundo digital provee.

\section{Revisión de las tecnologías en la industria 4.0}

El presente artículo aporta a la investigación un análisis de la contextualización de la era de la 
digitalización, en concreto se describe el impacto en las empresas, en las industrias 4.0 y cómo esta evolución tecnológica ha ocasionado cambios en los estándares y componentes de las industrias 4.0 (Rozo, 2020). La cuarta revolución industrial ha estado marcada por características relacionadas con tecnologías potencialmente innovadoras en las empresas fusionando los sistemas físicos, digitales y biológicos. En efecto, es importante la creación de redes de producción inteligente donde existe una interacción y colaboración entre sí, con el mundo digital y físico.

En definitiva, se ha evidenciado que el factor predominante en las revoluciones industriales es la innovación, la evolución en los cambios tecnológicos describen la digitalización de los procesos de las empresas como una interconexión mediante internet de las cosas, todo esto en particular con el fin flexibilizar la individualización de los procesos de las empresas, aunque todas las tecnologías avanzadas dan soluciones más flexibles e inteligentes, en particular siempre se trata de automatizar las empresas tecnológicamente con el fin de suplir las necesidades del medio.

En este estudio se lleva a cabo un análisis crítico de los impactos tanto positivos como negativos que posee las industrias 4.0, sin embargo, cada uno de los frentes tanto económicos, sociales y de ambiente tienen impactos negativos como positivos que ayudan a implementar la capacidad de adaptarse a nuevos cambios; todo lo anterior para mejorar los procesos internos de las empresas, el impacto en la economía y fomentar una mejor calidad de vida.

\section{Discusión}

Cada día la relevancia es cada vez más fugaz, la competencia es rigurosa, la innovación es rápida, y la Cuarta Revolución Industrial acelera todo esto. Entonces, ¿qué acciones debe tomar una organización? Hay gran cantidad de modelos para anticipar y responder a cambios de todo tipo, pero es necesario analizarlo desde una posición de riesgo. El riesgo no significa que algo sucederá, especialmente si toma medidas atenuantes.

El mayor riesgo para una organización en la Cuarta Revolución Industrial es que se vuelva irrelevante y no se adapte a las nuevas tecnologías y por consiguiente desaparezca. Y cuando se vuelve irrelevante, ya no puede existir. Se debe considerar reducir ese riesgo, para hacer esto, se sugiere el proceso de cuatro pasos: Uno, reunir inteligencia. Dos, evaluar la situación. Tres, evolucionar la estrategia. Y cuatro, de forma regular.

Reuniendo inteligencia, esto puede tomar varias formas; pero en su forma más básica se trata de educación. Pregunte, ¿entienden los líderes y otras partes interesadas de una industria la dinámica de la Cuarta Revolución Industrial? Y cuando lo hacen, ¿entienden los factores de riesgo generales? Leer artículos y libros, escuchar podcasts, mirar videos, consultar con compañeros, participar en conferencias, todos estos son mecanismos apropiados para elevar la inteligencia sobre este tema complejo.

En otras palabras, ¿cuáles son los riesgos específicos para la organización y la industria y cuáles son los riesgos de no actuar? Según la evaluación de riesgos, es probable que la estrategia de una organización deba cambiar. La idea de que una estrategia es buena durante varios años, generalmente de tres a cinco. No obstante, la mayoría de las organizaciones repiten su estrategia con frecuencia. El cambio constante y la falta de previsibilidad se convertirán en una característica común de la Cuarta Revolución Industrial.

La interrupción ocurrirá repentinamente y con frecuencia será desorientadora. La evolución de la estrategia puede ser suficiente a medida que cambian las condiciones, pero a veces puede ser necesaria una actualización completa de la estrategia. Esta nueva era se define por un mayor impacto, es decir, sin una idea, nuestro posible futuro no puede ser entendido. Y si las organizaciones no desarrollan su estrategia, 
el mundo cambiará demasiado rápido a su alrededor, dejándolas en el olvido. Las organizaciones existentes deberán cambiar, eso es un hecho. Pero para los nuevos participantes, el futuro puede ser más prometedor. A medida que las industrias y las organizaciones se vuelvan irrelevantes, surgirán otras nuevas. La Cuarta Revolución Industrial creará nuevas oportunidades increíbles para innovar como mínimo, hay que estar de acuerdo en que las organizaciones actuales no pueden simplemente superar esto. Los riesgos son demasiado altos, por lo anterior, se debe tener un plan y tomar las medidas, de lo contrario, la irrelevancia espera.

Entonces, que demuestra todo esto ¿estamos preparados?, ¿están las organizaciones preparadas a largo plazo en la implementación de la tecnología en sus procesos? Para determinarlo se necesita investigar casos de éxito, investigaciones, artículos que se presentaron en otras partes para así tomar en cuenta el impacto de las industrias $4.0 \mathrm{y}$ de esta manera adoptar medidas o estrategias que fortalezcan su incorporación en las organización con el fin de alcanzar una transformación digital con componentes adecuados a la realidad.

Como se ha visto anteriormente en el desarrollo de la investigación, todo lo anterior hace referencia a componentes que se encuentran dentro de las industrias 4.0 que ayudan a mejorar la transformación digital de las empresas con el objetivo de mejorar su competitividad. Por tal razón, el presente artículo busca analizar una posible implementación de la industria 4.0 como componente digital con el fin alcanzar una transformación digital en las pymes cucuteñas. Es importante mencionar que en la actualidad la pandemia que afecta al mundo y por ende muchos sectores de la economía; pero, aunque la crisis ha golpeado a la mayoría de sectores, la agroindustria y las industrias 4.0 (las TIC, canales de pagos digitales, comercio electrónico, entre otros) se han posicionado en medio del aislamiento obligatorio, teniendo un crecimiento del 3\% (Cámara de Comercio de Cúcuta, 2020).
La cuarta revolución industrial es inteligente, ¿qué es lo que quiero decir con eso? Mientras las revoluciones industriales anteriores estaban basadas, sobre todo en procesos, energía, productividad o materiales, esta se centra en la información constante y la respuesta automatizada. En el departamento de Norte de Santander hay un aproximado de 650 empresas de base tecnológica que han implementado en sus procesos las industrias 4.0, todas se encuentran registradas en la Cámara de Comercio de Cúcuta, están desarrollando estrategias digitales, aplicaciones, marketing y comercialización virtual (Diario La opinión, 2018). El departamento ha adoptado nuevos modelos de negocio, desde el 2016 existía la iniciativa entre la cámara de comercio de Cúcuta y la Universidad Francisco de Paula Santander (UFPS) de crear un clúster para las industrias a base tecnológica. No obstante la UFPS desde sus distintos programas se ha encargado de concientizar sobre el tema y los beneficios que trae para la economía es por esto que el objeto de estudio es el análisis y la implementación de las industrias 4.0 para así generar estrategias potenciales que favorezcan la transformación digital.

\section{Conclusión}

El presente artículo aborda temáticas de implicación de las industrias 4.0 y su aplicación como componente digital en las pymes cucuteñas. En primer lugar, se inicia con una explicación de lo que se entiende por cuarta revolución industrial, sus benéficos, componentes y los resultados que aporta a la transformación digital de la economía; por último, se analiza las amenazas que afronta en su implementación y qué estrategias son favorables, en respuesta a su aplicación. En conclusión, los retos que vivimos todo el tiempo nos obligan a un cambio constante (Drucker, 2005) que es lo que se analiza en el presente trabajo, un paso a la transformación digital de la economía.

En definitiva, las revoluciones tecnológicas impulsan los desarrollos más importantes de los seres humanos. Actualmente se está iniciando 
la Cuarta Revolución que está transformando la realidad que conocemos de una manera radical y a mayor escala que nunca antes. El futuro en el que todo esté conectado a Internet produciendo datos y tomando decisiones autómatas, ya se está presentando, aunque los desafíos medioambientales, sociales y económicos son muchos, por ende, es importante la revisión bibliográfica pertinente con el objetivo de adoptar estrategias en su adopción en las organizaciones.

Se determina que la Cuarta Revolución Industrial está cambiando la realidad que conocemos de manera radical y a mayor escala que nunca antes. Por lo anterior, es importante que las organizaciones se adapten desde ahora a la transformación digital. Como se ha mencionado anteriormente, si las empresas no piensan en fortalecer sus procesos de evolución ante los cambios y exigencias del mercado; si las organizaciones solo se centran en su capacidad de innovar a corto plazo, no será posible su éxito a largo plazo; en relación con la contratación de su capital humano, si la estrategia trata de resolver solo problemas en el presente y en un futuro se llegue el momento de resolver los obstáculos y problemas, las organizaciones podrán motivar el compromiso de su equipo con el desarrollo, crecimiento y competitividad de las empresas.

\section{Referencias}

Abdallah Alsaad, Abdallah Taamne. (2018). The effect of international pressures on the crossnational diffusion of business-to-business e-commerce. School of Business, Jadara University, 733, Irbid, Jordan

Alvar Villegas, J. (2017). Digitalización y mercados de exportación. Información Comercial Española, ICE: Revista de Economía, 898, 35-46. https:// doi.org/10.32796/ice.2017.898.1959 digital: Transformación de dimensiones, experiencias y modelos empresariales. Arbor, 188(754), 395-407. https://doi.org/10.3989/ arbor.2012.754n2011

Ana L. Abelianskya, Martin Hilbertb. (2016). Digital technology and international trade: Is it the quantity of subscriptions or the quality of data speed that matters?. University of Göttingen, Department of Economics, Germany

Ballestero, F., \& Pérez, M. (2017). Digitalización De La Economía . Estrategia Digital Y Políticas. 113-130.

Bekkers, E., Koopman, B., \& Teh, R. (2018). Long run trends in international trade. The impact of new technologies. 21st Annual Conference on Global Economic Analysis. https://www. gtap.agecon.purdue.edu/resources/res_display. asp?RecordID $=5562$

Bruno Moriset. (2020). e-Business and e-Commerc. University Jean Moulin, Lyon, France.

Boriana Rukanova, Stefan Henningsson, Helle Zinner Henriksen, Yao-Hua Tan. (2018). Digital Trade Infrastructures: A Framework for Analysis. Copenhagen Business School, Solbjerg, Denmark.

Buisán García, M., \& Valdés, F. (2017). La industria Conectada 4.0. Información Comercial Española, ICE: Revista de Economía, 898, 89-100. https:// doi.org/10.32796/ice.2017.898.1963

CAROLINE FREUND AND DIANA WEINHOLD. (2002). The Internet and International Trade in Services. American Economic Review. p.236240.

Ciuriak, D., \& Ptashkina, M. (2018). The Digital Transformation and the Transformation of International Trade Acknowledgements

Álvarez, E. G., Sintas, J. L., \& Martínez, A. S. (2012). Retos y tendencias del ocio 
Acknowledgements. January, 48. http:// e15initiative.org/wp-content/uploads/2015/09/ RTA-Exchange-Digital-Trade-Ciuriak-andPtashkina-Final.pdf

Cliff Wymbs, (2000) "How e-commerce is transforming and internationalizing service industries", Journal of Services Marketing, Vol. 14 Issue: 6, pp.463-477,

Comercial, C. I., Dominguez, R., Carolina, D., Domínguez, R., \& Carolina, D. (2019). Pin Ponce Virginia Roxana.

Cuadrado, A. (2019). Utilización Del Machine Learning En La Industria 4.0. Universidad de Valladolid, 108.

Eddy Bekkers, Robert Koopman and Robert Teh. (2018). Long run trends in international trade. The impact of new. World Trade Organization.

Ewelina Lackaa, Hing Kai Chanb, , Xiaojun Wangc. (2020). Technological advancements and B2B international trade: $\mathrm{A}$ bibliometric analysis and review of industrial marketing research. University of Edinburgh, Business School, UK.

Freund, C., \& Weinhold, D. (2002). The Internet and International Trade in Services. American Economic Review, 92(2), 236-240. https://doi. org/10.1257/000282802320189320

HE Yong, LI Jun-yang, WU Xue-pin, and JIANG Jiao-jiao. (2011) Impact of e-Commerce on International Trade-Based on a Iceberg Cost Model. Revista Internacional de Comercio, Economía y Finanzas.

Hernández Sampieri, Roberto; Baptista Lucio, P., \& Fernández Collado, C. (2014). Metodología de la Investigación. McGraw-Hill Interamericana, 533.

Industriales, E. D. E. I. (2019). Estudio de las aplicaciones de Machine Learning y Deep Learning en el ámbito de la logística y la fabricación.

Katz, R. L. (2017). El Observatorio De La Economía Digital De Colombia. MinTic, 1-44. https://bibliotecadigital.ccb.org.co/bitstream/ handle/11520/19664/El observatorio de la economì digtal de Colombia.pdf? sequence $=1$

Mark D. Robbins. (2018). Internet, Industry and International Trade: Digital Tradability in Services.Journal of World Trade (2)52.

Mauricio Bouskela, Márcia Casseb, Silvia Bassi, Cristina De Luca, M. F. (2016). La ruta hacia las Smart Cities. Inter-American Development Bank, 1-148. https://publications.iadb.org/bitstream/ handle/11319/7743/La-ruta-hacia-las-smartcities-Migrando-de-una-gestion-tradicional-a-laciudad-inteligente.pdf? sequence $=1$

Nuray Terzia. (2011). The impact of e-commerce on international trade and employment. Marmara University, Turkey.

Pernías, P. A. (2017). Nuevos empleos, nuevas habilidades: ¿estamos preparando el talento para la Cuarta Revolución Industrial? ICE, Revista de Economía, 898, 59-72.

Roberto, H., Carlos, C., \& Baptista, P. (2014). Metodología de la investigación. http:// observatorio.epacartagena.gov.co/wpcontent/uploads/2017/08/metodologia-de-lainvestigacion-sexta-edicion.compressed.pdf

Rozo-García, F. (2020). Revisión de las tecnologías presentes en la industria 4.0. Revista UIS Ingenierías, 19(2), 177-191. https://doi. org/10.18273/revuin.v19n2-2020019.

Shamel Azmeh, Christopher Foster. (2019).The International Trade Regime and the Quest for Free Digital Trade.The University of Manchester 
Susan Lund, James Manyika. (2016). Strengthening the global trade and investment system for sustainable development. McKinsey \& Company.

Suscriptores, N. D. E. (2018). ÍNDICE DE PENETRACIÓN A INTERNET. 4, 2018. 